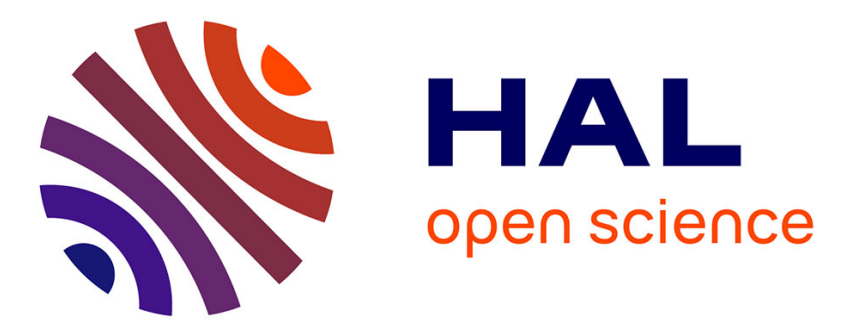

\title{
The ecology of heterogeneity: soil bacterial communities and $\mathrm{C}$ dynamics
}

Naoise Nunan, Hannes Schmidt, Xavier Raynaud

\section{To cite this version:}

Naoise Nunan, Hannes Schmidt, Xavier Raynaud. The ecology of heterogeneity: soil bacterial communities and C dynamics. Philosophical Transactions of the Royal Society B: Biological Sciences, 2020, 375 (1798), 10.1098/rstb.2019.0249 . hal-02895829

\section{HAL Id: hal-02895829 \\ https://hal.science/hal-02895829}

Submitted on 20 Aug 2020

HAL is a multi-disciplinary open access archive for the deposit and dissemination of scientific research documents, whether they are published or not. The documents may come from teaching and research institutions in France or abroad, or from public or private research centers.
L'archive ouverte pluridisciplinaire $\mathbf{H A L}$, est destinée au dépôt et à la diffusion de documents scientifiques de niveau recherche, publiés ou non, émanant des établissements d'enseignement et de recherche français ou étrangers, des laboratoires publics ou privés. 
Title: The ecology of heterogeneity: soil bacterial communities and $\mathrm{C}$ dynamics

Naoise Nunan ${ }^{1}$, Hannes Schmidt ${ }^{2}$, Xavier Raynaud ${ }^{1}$

5

${ }^{1}$ Sorbonne Université, CNRS, IRD, INRA, P7, UPEC, Institute of Ecology and Environmental Sciences - Paris, 4 place Jussieu, 75005 Paris, France

${ }^{2}$ Department of Microbiology and Ecosystem Science, University of Vienna, Vienna 1090, Austria

10

Correspondence: Naoise Nunan, naoise.nunan@upmc.fr

15

Keywords: heterogeneity, spatial modelling, degradation pathways, trade-offs, soil C dynamics

Statement:

20

The code for the spatial model can be downloaded from https://github.com/xraynaud/DivSpaceFunct. The NCBI taxonomy UID and the accession numbers of the bacteria can be found in the supplementary materials.

This is the accepted author manuscript (AMM)

25

30 


\section{Introduction}

In the last 10 to 20 years, soil microbial ecology has flourished, in no small part because of the development of molecular tools that have revealed the exceptionally large microbial diversity found in soils, e.g. [1]. These methodological developments led to a flurry of activity investigating the relationship between microbial diversity and many soil functions, such as the decomposition of organic matter or transformations in many elemental cycles e.g. [2]. However, Prosser et al. [3] cautioned that microbial ecology would not benefit from the accumulation of large amounts of data on microbial community diversity and composition unless it was couched in a theoretical ecological

45 framework. A number of attempts have been made to understand the relationship between microbial communities and decomposition of organic matter using ecological concepts borrowed from other branches of ecology. For example, microbial communities have been represented as specialist or generalist consumers [4] or $\mathrm{r}$ - and K-strategists [5], where it is assumed that there is a trade-off between the rate at which decomposers consume substrate and their ability to consume different substrates and to produce biomass, i.e. the yield [5-7]. Trait-based approaches, in which trade-offs among traits are allowed for, have also been used to provide evidence of links between microbial strategies, such as resource acquisition or biomass yield strategies, and organic matter decomposition $[8,9]$. Such analyses provide greater insight into the effects microbial properties can have on decomposition than simple measurements of microbial composition or diversity. Furthermore, they provide direction for future research. However, they all have in common the fact that they are organism centred. The effects of heterogeneous local environmental properties on microbial activity are conspicuous by their absence. In view of the fact that soil microbial communities are highly exposed and intimately linked to variations in their local environment [10,11], we suggest that this is a major omission. Heterogeneity is a fundamental property of soil that is often overlooked in

60 microbial ecology. Although it is generally accepted that the heterogeneity of soil underpins the emergence and maintenance of microbial diversity [12], the profound and far reaching consequences that heterogeneity can have on many aspects of microbial ecology and activity have yet to be fully apprehended and have not been fully integrated into our understanding of microbial functioning.

In the following we will first argue that the heterogeneity of the soil microbial environment,

65 and the consequent uncertainty associated with acquiring resources, affect how microbial metabolism, motility and interactions have evolved and, ultimately, affect the overall microbial activity that is represented in ecosystem models, such as heterotrophic decomposition or respiration. We will then present an analysis of predicted metabolic pathways for soil bacteria, downloaded from the MetaCyc pathway/genome database collection (https://metacyc.org/). The analysis suggests that, while there is

70 a relationship between phylogenic affiliation and the catabolic range of soil bacterial taxa, there does not appear to be a trade-off between the 16S rRNA gene copy number, taken as a proxy for potential 
growth rate, of bacterial strains and the range of substrates that can be used. Finally, we will present a simple, spatially explicit model that can be used to understand how the interactions between decomposers and environmental heterogeneity affects bacterial decomposition of organic matter.

\section{Direct effects of physical heterogeneity on microbial interactions and diversity}

Soil is a highly heterogenous medium, consisting of a mixture of solid material, of water-filled and of air-filled pores, all of which results in a wide range of micro-habitats, co-existing in close proximity [13]. The environmental properties and resource availabilities of the micro-habitats are often sufficiently different one from the other, that they constitute different ecological niches and can therefore harbour microorganisms, with different competitive abilities or life strategies, in relative isolation from one another $[12,14,15]$. Discontinuities in the water film, which place limitations on the extent of substrate diffusive fluxes also reduce the strength of the competitive interactions among individuals, as individuals don't compete for all the available substrate but rather use only that within their own diffusible domain [16]. The non-competitive diversity patterns that are so characteristic of soils [17] are therefore believed to stem from the physical constraints and heterogeneity of the microbial environment that limit direct competition among taxa and individuals and therefore reduce competitive exclusion [18].

\section{Metabolic costs of resource acquisition and adaptations}

Heterotrophic bacteria satisfy their resource requirement from outside the cell. They generally do this by secreting extracellular enzymes into their surroundings, to which significant metabolic costs, both energetic and elemental, are associated. Before discussing the effects that heterogeneity might have on resource acquisition, we will look at the costs of resource acquisition and how these are minimised by bacteria.

Protein synthesis, including the synthesis of extracellular enzymes, accounts for approximately $75 \%$ of a bacterial cell's energy expenditure [19]. The energetic burden of a cell's protein synthesis is such that the synthesis of a given protein is not likely to survive selective pressures unless it confers an increase in fitness [20] and, indeed, it has been shown that the growth of bacterial cells is hindered when they synthesize proteins unnecessarily [21]. Furthermore, once secreted, extracellular enzymes are generally lost to the cell because they tend not to be able to retrieve the secreted enzymes (due to a lack protein import systems). As a result, extracellular proteins are not recycled by the cell, as is the case for intra-cellular proteins. In order to maximise fitness, micro-organisms minimise the metabolic costs associated with the secretion of enzymes.

The cost minimisation strategies are evident in the deployment of a range of phenotypic adaptations. The energetic cost of extracellular enzymes is generally lower than that of intra-cellular 
enzymes because they tend to contain fewer amino acids that are metabolically expensive to produce [22]. The energy required to synthesise individual amino acids varies quite considerably, spanning almost an order of magnitude in E. coli [23]. The substitution of costly amino acids with less costly 110 ones in extracellular proteins can reduce the cell's energetic budget [22]. It has also been shown that the elemental composition of enzymes involved in the acquisition of $\mathrm{C}$ and $\mathrm{S}$, enzymes that are often expressed in response to shortages in $\mathrm{C}$ and $\mathrm{S}$, are depleted in these very elements [24], thus reducing the metabolic cost to the cell. The expression levels of proteins change to maximise growth in the cost:benefit environment (i.e. resource availability) in which microbes find themselves: levels of

115 induced expression are higher in environments with higher substrate concentrations and where the return on metabolic investment is higher [25].

There are two types of extracellular enzymes, free and membrane-attached, the production of which may also be optimised to suit the cost:benefit environment [26]. Free extracellular enzymes tend to catalyse the production of more product than membrane attached enzymes because substrateenzyme encounters tend to be more frequent [26]. However, the reactions do not necessarily occur in close proximity to the parent cell, due to the diffusion of the enzyme, meaning that much of the reaction product may not be of great benefit to the enzyme producer. This means that the cell must maintain a high rate of enzyme synthesis and secretion to compensate for diffusive loss. Free extracellular enzymes are therefore a cost-efficient resource acquisition strategy in copiotrophic environments, where substrates are in the micromolar range, and when the producer cell lives within a community, where other cells can benefit from the activity of the enzyme [26]. The production of such enzymes may be regulated by quorum sensing, so that production is only initiated when the community is likely to benefit [27]. Membrane-attached extracellular enzymes, on the other hand, are a more cost-efficient strategy for nutrient acquisition in oligotrophic environments, where substrate

130 concentrations are generally in the nanomolar range or lower. Membrane-attached extracellular enzymes may also be the strategy of choice for isolated cells. This strategy has the advantage of reducing the enzyme synthesis to product acquisition ratio, thus reducing the cost to the cell. As we will see below, soil is generally an oligotrophic environment, with concentrations of organic molecules in the soil solution in the nanomolar range [28,29]. Furthermore, outside of the rhizosphere,

135 soil bacterial cells tend to have relatively few neighbours [30], reducing the likelihood that other cells might profit from the products of their enzymatic activities.

\section{Adaptations to heterogeneous environments}

The strategies for minimising the metabolic cost associated with the acquisition of extra-cellular 140 resources are likely to be reinforced in heterogeneous environments such as soil, where the return on investment is highly uncertain. The uncertainty of the return on enzyme investment stems from the 
combination of the heterogeneous nature of the available resource and a spatially heterogeneous physical environment. As we will attempt to show in the following paragraphs, the interaction between resource heterogeneity and spatial heterogeneity reduces the probability of other strategies 145 of resource acquisition, such as motility or resource use at a community level, being employed. As a result, evolution may have pushed soil microbial decomposers towards a cautious approach to investment in resource acquisition.

The resources available to soil microbial communities are very heterogeneous in nature. The organic matter dissolved in the soil solution (that which is the most available for microbial use) is made up of a variable collection of molecular species, with several thousand different formulae being readily detectable [31]. If the environment were homogenous, then the cost of maintaining the enzymatic capacity necessary to consume such a heterogeneous substrate, although probably too great for a single microorganism [19], could be borne by the whole community. The community might then be made up of specialists, each consuming a limited range of substrate, thus reducing competition amongst members of the community and increasing complementarity [32].

On the other hand, if the substrate were homogenous but the environment spatially heterogeneous, then the substrate assimilation costs would be comparatively low as decomposers would only have to bear the cost of a rather limited number of enzymes. They could then expend more energy on strategies, such as motility, for exploring the heterogeneous space. This strategy is used by some decomposers in marine environments [33,34]. However, the energetic cost of motility, especially in the case of rapid swimming, is also high [33]. As a result, motility and chemotaxis are broadly confined to copiotrophs, whereas oligotrophs are mostly non-motile [34]. In soil, surface tension, capillary forces and viscous drag in the water-filled porosity [35] increase the energy required for motility and these physical constraints, particularly in partially saturated pore networks, are such 165 that any potential motility is confined to matric potentials in the wet range [36]. Motility was found to cease, virtually completely, when the thickness of the water-film reached $1.5 \mu \mathrm{m}$. Fungi also have the ability to explore the soil pore space, through hyphal spread rather than by swimming in the soil solution, and they are more active and have relatively more biomass in soil with high organic matter contents [37,38], corroborating the suggestion that there is a high energetic requirement for exploring 170 the soil pore space.

Where both resources and the spatial environment are heterogeneous, the supply of any particular resource, in any given location in the pore network, is likely to be low [28,29], due to the distribution of the resource throughout heterogeneous pore space. In this scenario, the probability of there being a sufficient quantity of substrate within the vicinity of a decomposer to make the 175 production of enzymes metabolically worthwhile is likely to be lowered significantly. It has been shown that low substrate concentrations constrain decomposition [39], which tends to confirm this 
view. Were this the case, then the idea that microbial decomposers forage for resources through the production of extracellular enzymes is improbable. The low substrate concentrations are also likely to limit motility as it has a high energy requirement.

If soil microbial decomposers find themselves in a harsh and unreliable local environment in which both resource acquisition and motility are constrained, what strategy should they use? Here, we suggest that the selective landscape that emerges from the interaction between resource and environmental heterogeneities has pushed microbial decomposers to "play a waiting game". This hypothesis is supported by the fact that many soil bacteria produce extracellular polymeric substances

185 [14]. Soil bacteria allocate resources to the production of extracellular polymeric substances (EPS), as an insurance against, or buffer for, fluctuating conditions in their environment [14]. For example, the production of EPS has been shown to maintain moisture levels of the microbial microenvironment [40]. Extracellular enzymes can be located within the EPS matrix in close proximity to cells [41], thus reducing diffusional loss of both the enzyme and the product and increasing the likelihood that

190 the products of the activity of these enzymes will benefit the cells. EPS may also decrease the diffusion rates of substrates towards the cell, but also acts as a trap for nutrients and substrate [42], which suggests that EPS producers may accept a lower potential acquisition rate for increased probability of resource acquisition. The energetic cost of EPS production to the cell is non-negligible [43] however, suggesting that this is a necessary adaptation to the environment in which the cells find 195 themselves.

The overall picture that emerges from these considerations is that soil bacteria have, for the most part, relatively passive resource acquisition strategies and that their activity depends on the arrival of resources rather than on active foraging for resources. It follows that the constraints imposed by the spatially heterogeneous resource landscape should be accounted for if the microbial roles in 200 ecosystem fluxes are to be fully understood.

\section{Metabolic traits of soil bacteria}

Microorganisms are characterised by a set of traits, their life history strategy, that will also have an impact on how $\mathrm{C}$ is processed by microbial communities [9]. As molecular heterogeneity is a

205 hallmark of soil organic matter [31], one of the traits most likely influence microbial processing of C is the catabolic breadth (the range of organic substrates that can be used) of an individual cell. If a decomposer cell does not have the capacity to consume a substrate, then the substrate will persist, regardless of its availability to the cell or its chemical properties. The greater the catabolic breadth of decomposers, the more likely substrate is to be consumed upon decomposer-substrate encounters.

210 The rapidity with which a decomposer cell responds to the presence of a given substrate and the physiological allocation of C (to cellular maintenance or growth) of the cell will also affect $\mathrm{C}$ cycling. 
The trade-offs among these traits determine the impact that each decomposer can have on the dynamics of $\mathrm{C}$ in soil. For instance, there may be a trade-off between the rate at which resources are acquired and the growth efficiency, or $\mathrm{C}$ use efficiency, of a cell: high investments in resource 215 acquisition reduce the growth efficiency of the cell [44]. Similarly, in environments with heterogeneous resources, adaptations to make use of multiple resources may have a negative impact on growth rates [8]. It should be borne in mind however, that the conditions in soil may limit the range of life histories strategies that can flourish.

\section{Bacterial catabolic breadth, growth rates and phylogeny}

In order to test whether there were indeed trade-offs between catabolic breadth, growth rate and carbon use efficiency, we analysed the predicted metabolic functions of a number of bacterial strains found in soils that were stored the MetaCyc database [45]. We determined the relationship between the number of degradation/utilisation/assimilation pathways and the 16S rRNA gene copy number for each bacterial strain. The 16S rRNA gene copy number was taken as a proxy of potential growth rate and carbon use efficiency [46]. We also determined the relationship between the catabolic range and the phylogenetic affiliation of the strains in order to determine whether 16S rRNA gene barcoding might be a useful proxy for life history strategies relevant to $\mathrm{C}$ cycling in soil.

The Metacyc database contains sequenced genomes that provide information on metabolic

230 function from all kingdoms of life. We extracted 843 records of soil bacterial genomes from the database. These 843 records contained 439 consolidated pathways for degradation/utilisation/assimilation of substrate. These pathways were further consolidated to 313 by grouping different pathways having similar roles (e.g. urea degradation I and II; see supplementary materials for all methodological details). In order to ensure that the bacterial strains in the database

235 were representative of soil bacteria, we first looked at their phylogenetic affiliation and found that the relative abundances of the major phylotypes (Fig. S1) were not fundamentally different from those of the dominant soil phylotypes from across the globe [47]. Actinobacteria and Proteobacteria were the major phylotypes in both datasets; Firmicutes and Bacteriodetes were slightly over-represented in the Metacyc database whilst Acidobacteria and Planctomycetes were under-represented. The

240 under-representation of Acidobacteria (only 24 Acidobacteria present in Metacyc, compared to more than 5000 Proteobacteria) was the main difference between the bacteria in the database and the dominant phylotypes from across the globe. This was probably due to the fact that Acidobacteria are notoriously difficult to isolate from soil [48].

Having established that the database was reasonably representative, we determined the 245 relationship between degradation/utilisation/assimilation pathways and phyla, using only strains from phyla for which there were at least 10 entries in our soil bacteria database. The number of pathways 
varied widely across the strains, ranging from 14 to 139 , and there were significant differences in the average number of pathways among phyla (between 45 and 70; Fig. 1). We also checked the number of pathways in strains from rarer phyla and found that the number of 250 degradation/utilisation/assimilation pathways in Acidobacteria, Chloroflexi and Planctomycetes was of the same order of magnitude. These data suggest that there are specialists, with few degradation/utilisation/assimilation pathways, and generalists, with many pathways, in all phyla and, despite the differences among phyla, that phylogenetic affiliation is not likely to be a good proxy for identifying generalist or specialist strategies, with respect to resource acquisition and utilisation at least. The large variations in the number of pathways within phyla is most likely a reflection of differences in evolutionary processes that occur in soil bacteria. In some cases there was less variation in the number of pathways within phylogenetic class, particularly within the Proteobacteria, but large variation still remained (Fig. S2). We did not analyse the data for differences among classes as many of the classes did not have a sufficient number of entries for statistically reliable results. Similarly, we did not have a sufficient number of entries to analyse the data at the order level. One should of course not lose sight of the fact that the Metacyc database is based on the sequenced genomes of isolated bacteria and therefore may give a biased picture, even though the relative abundances of phyla in the database appear to be reasonably representative. Furthermore, the pathways in the database are limited to those that are known and the true picture may very well be different to the one shown here.
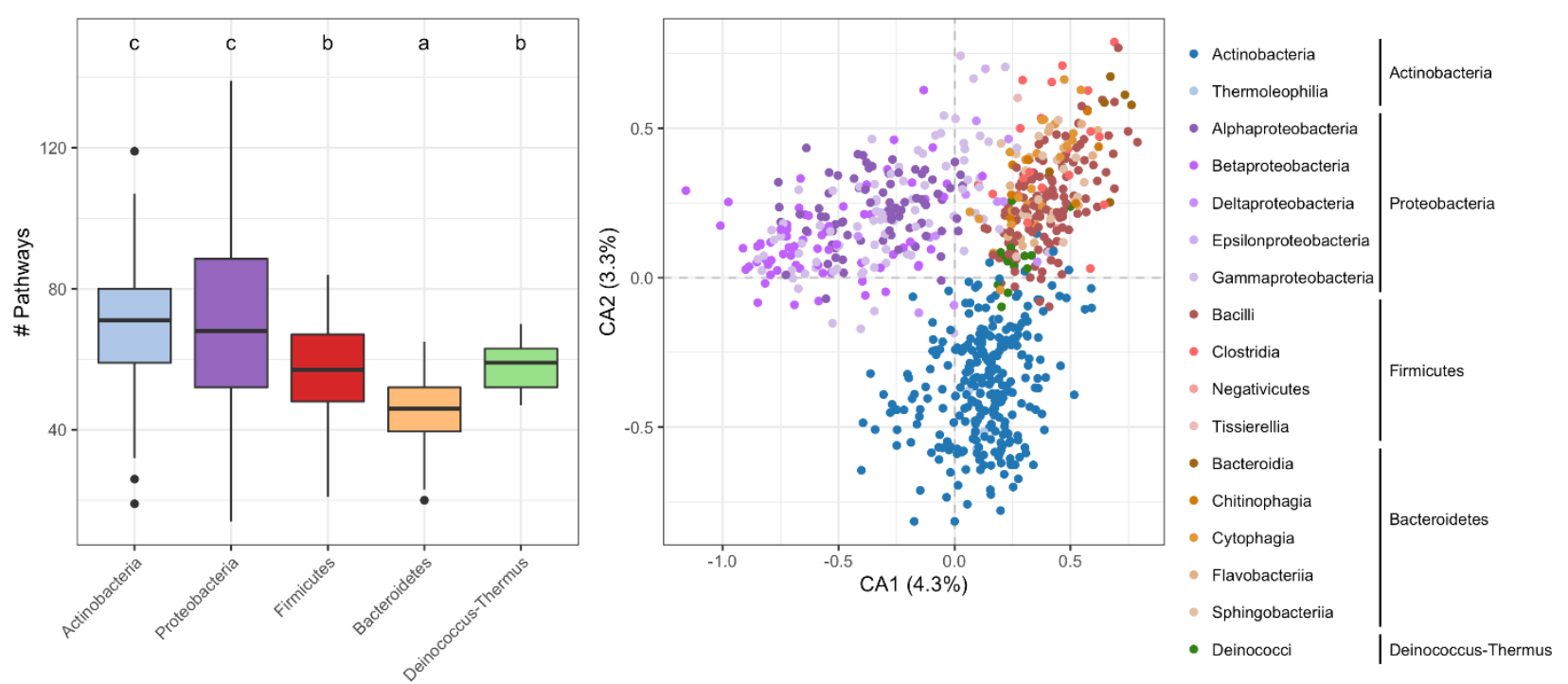

Figure 1 The number of pathways per strain, as a function of phylum (left panel) and the ordination plot of the correspondence analysis based on the presence or absence pathways in strains (right panel). The colours correspond to phyla and the shades to classes. In panel a, different letters indicate significant differences between phylum (GLM Poisson model). 
There were similarities in the types of pathways within phyla (Fig 1). Actinobacteria and

270 Proteobacteria in particular had slightly different sets of pathways compared to each other and to the other phyla. The fact that the degradation/utilisation/assimilation pathways are not distributed randomly across phyla may have consequences for $\mathrm{C}$ cycling. A number of studies have indicated that the co-occurrence of operational taxonomic units (OTUs) from the same phylum tends to be higher than one would expect from random associations [49]. Were OTUs from the same phyla also

275 to aggregate at fine scales (the microbial habitat scale for example), then the distribution of degradation/utilisation/assimilation pathways would be uneven across microbial habitats. It is conceivable therefore, that organic molecules not being co-located with the pathways required to decompose them may contribute to their persistence in soil. The extent to which this affects persistence would depend on how dynamic the soil structure is and therefore the rate at which 280 decomposers and organic molecules are redistributed in space.

There is a metabolic cost associated with maintaining a high number of metabolic pathways and one might expect there to be trade-offs that affect maximal growth rates (i.e. the maximal growth rate under nutrient replete conditions and without extrinsic constraints). This idea is often included in ecological models of microbial decomposition of substrate [6,8]. In order to check whether bacteria

285 exhibited trade-offs between maximal growth and degradation/utilisation/assimilation pathways, we analysed the relationship between the number of pathways and the 16S rRNA gene copy numbers of strains. It has been shown that the 16S rRNA gene copy number is related to both maximal growth rate and carbon use efficiency [46]. The 16S rRNA gene copy number for 228 of the soil bacteria present in the Metacyc database were retrieved from the rrnDB database [50] (see supplementary

290 methods for methodological information). No clear relationship was apparent between the number of pathways harboured by strains and the number of copies of the 16S rRNA gene across strains from all phyla (Fig 2). However, positive relationships were found within Proteobacteria and Firmicutes (Fig 2), also suggesting a lack of trade-off between catabolic breadth and maximal growth rate.

For a trade-off to be apparent in the genome signature of an organism, the organism must have 295 evolved in an environment that is favourable for the emergence of the trade-off. In environments that are characterised by trophic heterogeneity (as is the case for soil), one might expect to see a trade-off between catabolic breadth and growth. However, the conditions required for such a trade-off to emerge may not be constant enough in soil and, indeed, environmental fluctuations themselves may be a significant evolutionary driver. It has been suggested that the $16 \mathrm{~S}$ rRNA gene copy number in $E$.

300 coli is optimised to maximise fitness under the fluctuating conditions rather than growth in constant environments [51]. Furthermore, other constraints on growth (lack of resources, external stressors) imposed by the environment may also affect the relationships among traits and the relationship between traits and fitness. It has recently been shown that there is no relationship between the $16 \mathrm{~S}$ 
rRNA gene copy number and growth in soil under natural conditions [52]. However, a relationship between growth and the 16S rRNA gene copy number became apparent after the addition of abundant substrate, thus removing the resource constraint.

We cannot completely discount the possibility that a culture bias may have affected the results. For instance, if there were more representatives of the generally slow growing Acidobacteria a different relationship might have become apparent.

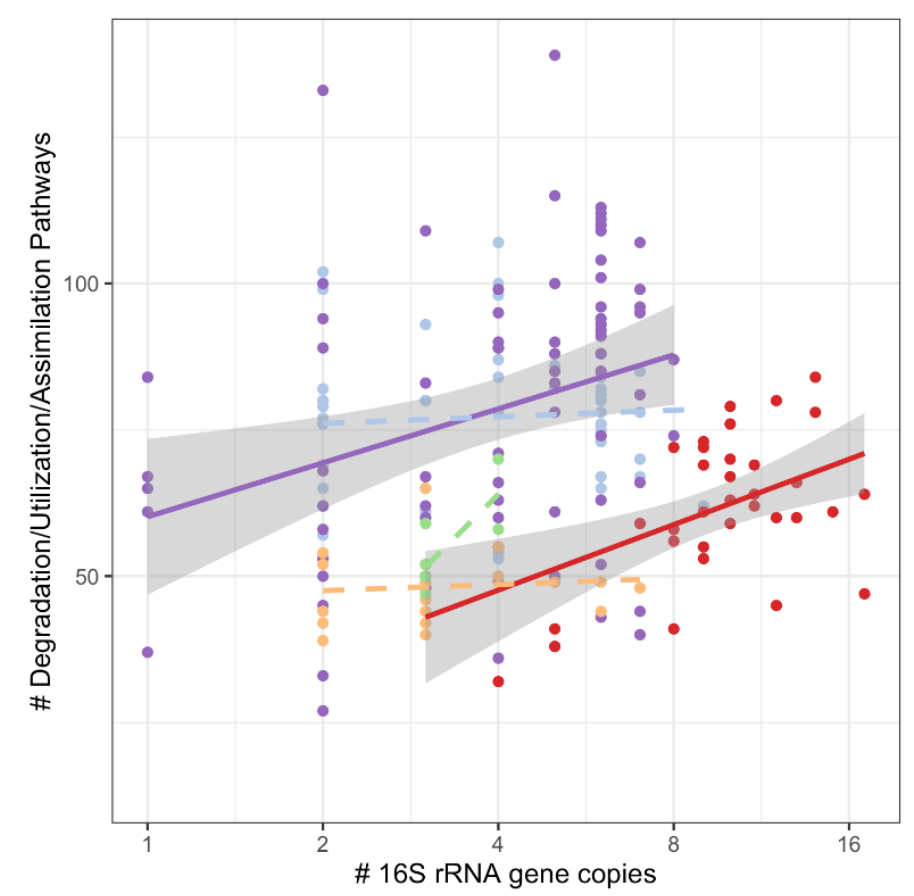

Figure 2 Number of degradation/assimilation pathways versus $16 S$ rRNA gene copy number for 228 strains that were in both the Biocyc and rrnDB databases. Colours represent different phyla (see Fig 1 for legend). Solid lines show significant relationships and dashed lines the non-significant ones.

\section{Spatial model to account for the interaction between heterogeneous resource availability and decomposer metabolic traits}

In view of the likely relative immobility of soil bacteria, especially in relation to substrate diffusion rates [36,53], it is reasonable to treat bacterial decomposers as spatial point processes. The intrinsic properties of the individual decomposers, such as catabolic breadth or growth rates, can be assigned to individual points of the point process, as can local habitat constraints, such as resource availability. We suggest that the role of microbial communities in $\mathrm{C}$ cycling can only be apprehended if the interaction between resource availability and decomposer metabolic traits is accounted for. Resource availability is a product of the interaction between the physical heterogeneity of soil and the molecular heterogeneity of soil organic matter and so is itself highly heterogeneous. The next section will explore how these interactions can affect $\mathrm{C}$ cycling. The aim of the analysis is not to 
provide a model of soil $\mathrm{C}$ dynamics - this is well beyond the scope of this study as it would require

325 upscaling and extensive parameterisation. The aim is rather to determine how heterogeneities in resource availability and distributions of bacterial resource acquisition strategies might affect decomposition.

We chose to use a simple, spatial modelling approach. The spatial model consisted of a $100 \mathrm{x}$ 100 pixel grid onto which were distributed $n$ different resources and 120 decomposer cells belonging

330 to $s$ distinct taxa. Assuming a pixel side of $2 \mu \mathrm{m}$, the cell density was equivalent to $3000 \mathrm{cells} / \mathrm{mm}^{2}$, which is approximately equivalent to $10^{9}$ bacterial cells $\mathrm{g}^{-1}$ soil [30], depending on the specific surface area of the soil. The parameters $n$ and $s$ both varied between 1 and 10. Each of the $n$ resource types were randomly distributed on the grid as discs of radius $R$. The total number of resource discs distributed on the grid was such that $50 \%$ of the grid cells contained resources. When the disc radius

$335 R$ was small, resources were evenly, but randomly, distributed and when $R$ was large, the distribution of resources was more aggregated.

Bacterial communities where represented in the model as planar marked point processes. Each cell was able to use from one to ten resources. Each decomposer cell, represented in the model as a single point, acquired resources within a disc of radius $r$ pixels. The parameter $r$ represents the access

340 decomposers have to the resources and it depends on both the heterogeneity of the environment and the ability of the decomposers to acquire the resources. The more heterogeneous the environment and the more disconnected the water films in the pore space, the smaller the radius of the disc, as the diffusion of resources to the decomposer cells is restricted. The radius of the disc also increases as a function of the rate at which resources are consumed by a decomposer. The faster a resource is 345 consumed the steeper the concentration gradient around the decomposer and the greater the diffusion. Therefore, in a given environment, a more competitive decomposer would have a larger disc than a less competitive decomposer. For simplicity's sake, the cells consumed all resources available to them within the disc immediately, if they had the capacity to consume them.

Depending on the simulation, decomposer cells of a single taxon were either randomly 350 distributed across the grid or aggregated. The total area from which all decomposers of a single acquired resources (i.e. the union of all discs of radius $r$ surrounding the cells of the taxon) is a GermGrain model [54]. In the case of random distributions this area is a Boolean model [55], and is given by its coverage function. $c(s, r)=0.5 * 1-e^{-\lambda_{s} \pi r^{2}}$ where $\lambda_{s}$ is the intensity of the point process for taxon $s$ (i.e. the number of cells of taxon $s$ per unit area). Therefore, as the $50 \%$ of the grid cells 355 always contained resources, the expected proportion of resources acquired by taxon $s$ is

$$
p_{s}=0.5 \times c(s, r)=0.5 * 1-e^{-\lambda_{s} \pi r^{2}}
$$

In order to determine how the spatial distribution of decomposer cells might affect resource use, we also simulated decomposer cells with aggregated distributions using a clustered Matèrn distribution 
[56]. Cell were grouped into 10 clusters, themselves distributed randomly across the grid. Within each

360 cluster of radius $r_{c}$, cells were randomly distributed. The value of $r_{c}$ determined the extent of aggregation (10px: highly aggregated, 25px: slightly aggregated). In order to allow for comparison between random and aggregated distributions, we forced the aggregated distributions to have the same number of cells (120) as the random distributions of cells, by drawing distributions until we obtained the desired number of points. Cells were then randomly attributed to taxa. In contrast to the

365 Boolean model, the coverage function of a Matèrn distribution is not known.

The effects of sets of parameters describing the spatial distribution of both resources and decomposers on resource use was tested by running 100 simulations in each case. The results are presented as boxplots in the different figures.

\section{Effects of environmental heterogeneity}

Figure 3 shows how the proportion of total resource consumed varies as a function of the resource patch size and as a function of the access that decomposers have to resources (the area of the environment that decomposers can explore, which is related to the heterogeneity of the environment and represented by the disc of radius $r$ ). In these simulations there were 10 different

375 resources and 10 different bacterial taxa, each of which could consume only one resource. The proportion of resource consumed increased significantly as the access to resources increased. This means that the more homogenous and the more connected the environment, the more resource will be consumed. This is easily translated to the situation in soil: it is well known that organic matter is decomposed more rapidly in sandy soils, where organic matter and decomposers are less segregated,

380 than in soils with higher clay contents [57]. In sandy soils the total surface area is lower than in clay soils, meaning that the probability of contact between substrate and decomposer is higher. The size of the resource patches did not affect the average amount of resource consumed when the area within which decomposers acquire resources remained constant, but the variance increased as the resource patches increased in size (Fig 3). It should be noted that when the size of the resource patches were smaller than the area explored by individual decomposers, the variance was quite small; when the resource patch sizes were larger than the area explored by individual decomposers, the variance increased quite abruptly. This can be explained as follows: if resources are available as many of small patches, then these will be widely distributed and the probability of cells being in contact with resource patches is high. In contrast, if resources are aggregated in larger patches, then the likelihood of cells having access to either large amounts of resources or to none will be high (Fig S3). 


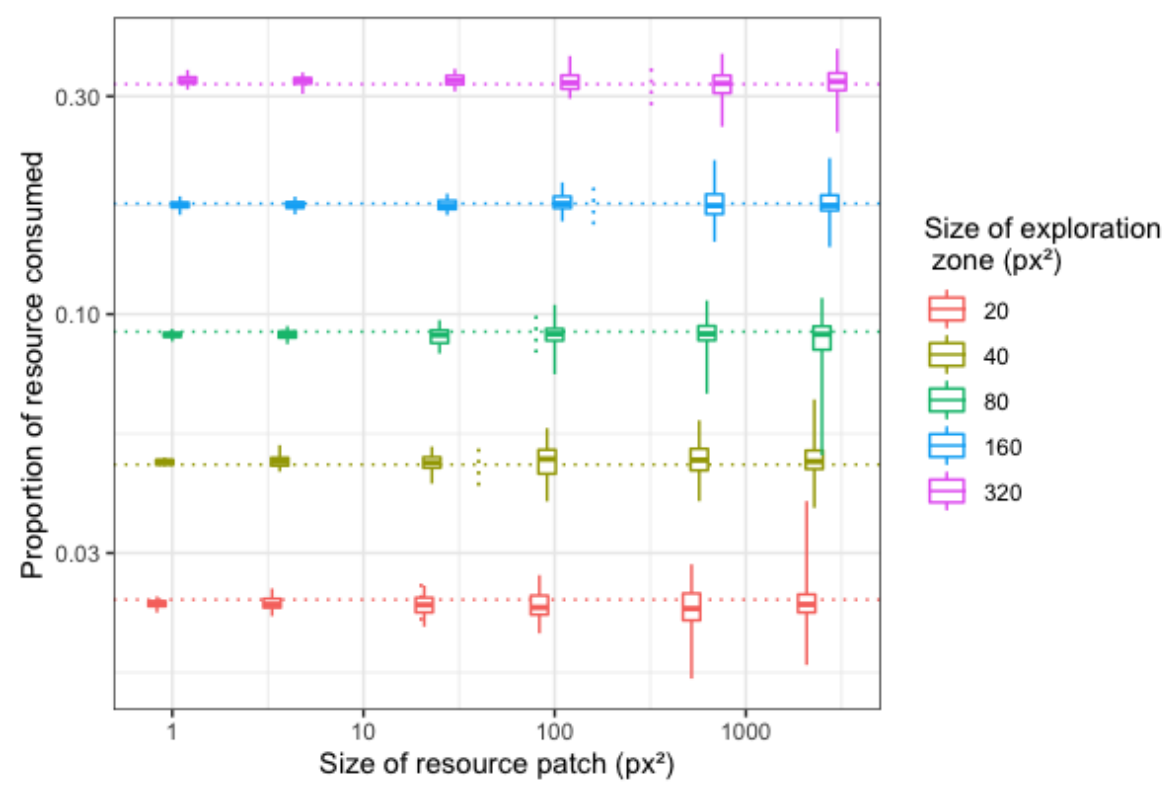

Figure 3 Proportion of resource consumed by a community as a function of the radius of the disc within which cells acquire resources and the spatial distribution of resources. The area of the discs ranged from 20 to 320 px2. Horizontal lines correspond to the theoretical values for the proportion of resource consumed (Eq. 1). Vertical dotted lines indicate the size of the area within which cells acquire resources. Note that the scales of both axes are logarithmic.

395

\section{Effects of decomposer community properties}

In Figure 4 we show how changes in decomposer diversity can affect decomposition. Here, the total number of decomposer cells (120) and the area within which they acquired resources $(r=40 \mathrm{px})$ remained constant across the $100 \times 100$ px grid. In these simulations, each taxon could consume only one resource type. The resources were distributed in patches as described above, with resource patches varying in size between 4 and $625 \mathrm{px}^{2}$. The simulations were carried out with $1,2,4,8$ or 10 taxa, meaning that the community could consume $1,2,48$ or 10 types of resource. The number of cells per taxon was calculated as 120/(number of taxa), i.e. the total biomass was constant. Resource use increased with increasing diversity, i.e. more taxa with fewer individuals consumed more resource than fewer taxa with more individuals. This is in accordance with non-spatial modelling results [4]. However, it should be noted that as the resource patch sizes increased, the resource use variance also increased. The interaction between functional diversity (number of resources a community can use) and resource distribution could result in less diverse communities using more resource than more diverse communities, despite lacking the capacity to use part of the resources.

The analysis of the Metacyc data showed that there were wide variations in number of degradation/utilisation/assimilation pathways among strains, indicating that both specialists and generalists are widespread, regardless of phylogenetic affiliation (Fig. 1 \& Fig. S2). We explored the effect that the different resource acquisition strategies might have on resource use in heterogeneous environments by running simulations in which 120 cells belonging to 1, 2, 5 and 10 different taxa, were able to use, respectively, 10 (full generalist), 5, 2 or 1 (full specialist) different types of resource. 
Although the analysis of the Metacyc data suggests that there is little or no trade-off between the degree of generalism (catabolic breadth) and potential growth, we also looked at how such a tradeoff (were it to exist) might affect resource use. As the model does not explicitly represent growth or resource use efficiency, this was achieved by varying the radius of the disc within which decomposer cells acquired resources as an inverse function of the catabolic breadth. This was felt to be an appropriate way to represent specialists and generalists as one would expect specialists to consume resources more rapidly than generalists, through the deployment of more or more efficient transporters for example, thus creating steeper resource gradients around the decomposer and extending the diffusion lengths. The radius ( $r$ ) of the disc within which cells acquired resources was

425 therefore reduced as a function of the number of resources a taxon could use. This was termed the trade-off value. Three trade-off values were considered: no trade-off ( $\mathrm{r}=$ constant), moderate tradeoff $(r=$ constant $/ \sqrt{\# \text { of resource used }})$ and high trade-off $(r=$ constant $/ \#$ of resource used $)$.

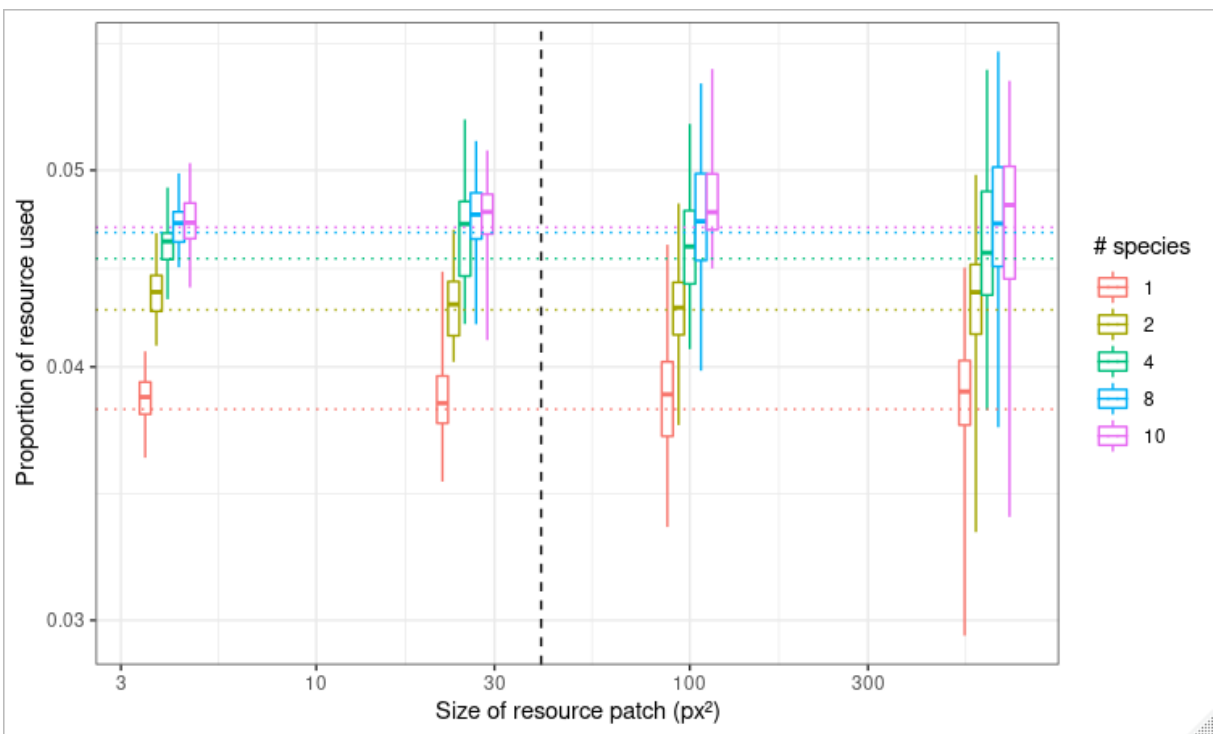

Figure 4 Proportion of resource consumed by a community as a function of microbial diversity and the spatial distribution of the resources. All decomposers acquired resources from within a disc with a radius of 40px and decomposer biomass was constant (120 cells in the $100 x 100$ grid). There were 10 resources available and each decomposer could use only one resource. Note that the scales of both axes are logarithmic.

In the absence of trade-offs, all else being equal, generalists use the available resources more completely, conferring them a competitive advantage over specialists (Fig 5, left panel). This is because the probability that a generalist encounters a resource it is capable of using is higher than the probability of a specialist encountering the specific resource that it can use. However, the picture 435 changed when the potential trade-offs were accounted for (Fig. 5, centre and right panels). Depending on how large the trade-off is, generalists may even be less competitive than specialists. As with previous simulations, increases in patch size resulted in higher variance in the proportion of resource consumed, regardless of the resource acquisition strategy. 
It should be noted that the 10 species communities in Figures $4 \& 5$ are the same (i.e. communities of specialists) and the proportion of resource consumed is identical. However, the single species communities in Figure 4 are also specialists whilst they are generalists in Figure 5. The patterns in Figure 4 show the effect of an increase of functional diversity (increase in the number of resources that can be used) within a community, whilst the patterns in Figure 5 (left panel) show the effect of an increase in the spatial coverage of the functional diversity, but without any changes to the 445 functional diversity itself.

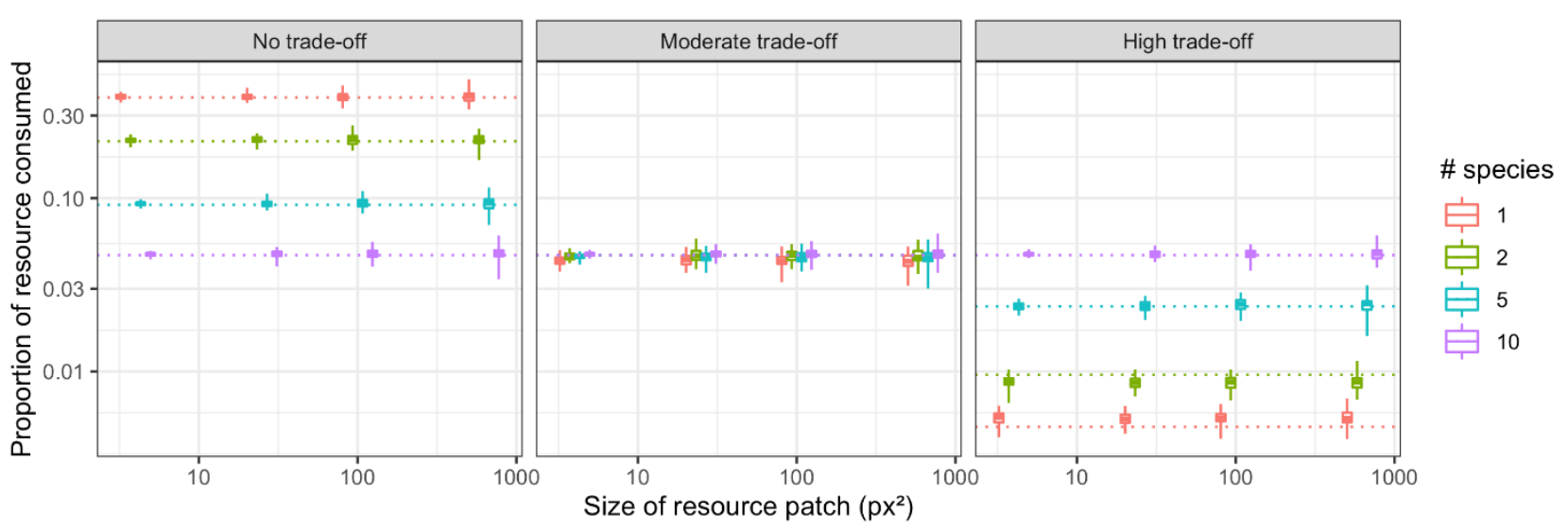

Figure 5 Proportion of resource consumed by a community as a function of the number of resources a decomposer can use and the spatial distribution of the resources (within each panel). The effect of the trade-off related to the cost of multiple resource use is shown in the different panels (from left to right: no trade-off, moderate trade-off and large trade-off). There were 10 different resources. When there were 10 taxa, each could only use a single resource (specialists) and when there was only one taxon, it could use all 10 resources (generalist). In the no trade-off panel, all bacteria acquire resources from within a disc with a radius of $42 \mathrm{px}$. In the moderate trade-off panel, cells acquired

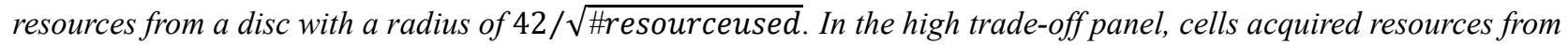
a disc with a radius of 42 / \#resources used. Note that the scales of both axes are logarithmic. The slight deviations from the expected proportions (dashed lines) that were sometimes observed are due to discretization in the model.

\section{Distribution of decomposer communities}

The analysis of the decomposition/utilisation/assimilation pathways showed that some phyla (Proteobacteria and Actinobacteria) tended to have more pathways than other phyla, suggesting a broader resource use capacity (Fig. 1). These phyla also tended to have a slightly different resource use profile. We therefore gave decomposers different resource use capacities and resource use profiles in order to mimic the resource use capacities and profiles shown in Figure 1: two decomposer taxa could use three resources (i.e. Proteobacteria and Actinobacteria), two could use two resources (Firmicutes and Deinococcus-Thermus) and one could use only one resource (Bacteriodetes). As with the other simulations, there were 10 different resources available to the decomposers on the grid. All the decomposers that could use three resources used one common resource; the other two resources they could use were assigned randomly. All other decomposers were assigned resource uses at random. Figure 6 shows that when resource use was distributed in this way across the decomposer 
community and the decomposers were distributed randomly in space, the proportion of total resource used was greater than when each decomposer could only use one resource (Fig. 4), but smaller than when decomposers were generalists without trade-offs (i.e. could use all 10 resources; Fig. 5, left panel). The picture may be different if there were trade-offs related to the cost of multiple resource use (Fig. 5, centre and right panels). Resource use decreased and became far more variable as the distribution of decomposers became more aggregated (Fig. 6). The dramatic increase in resource use variability with higher decomposer aggregation is reminiscent of the hotspots of microbial activity that are believed to be prevalent in soils [58].

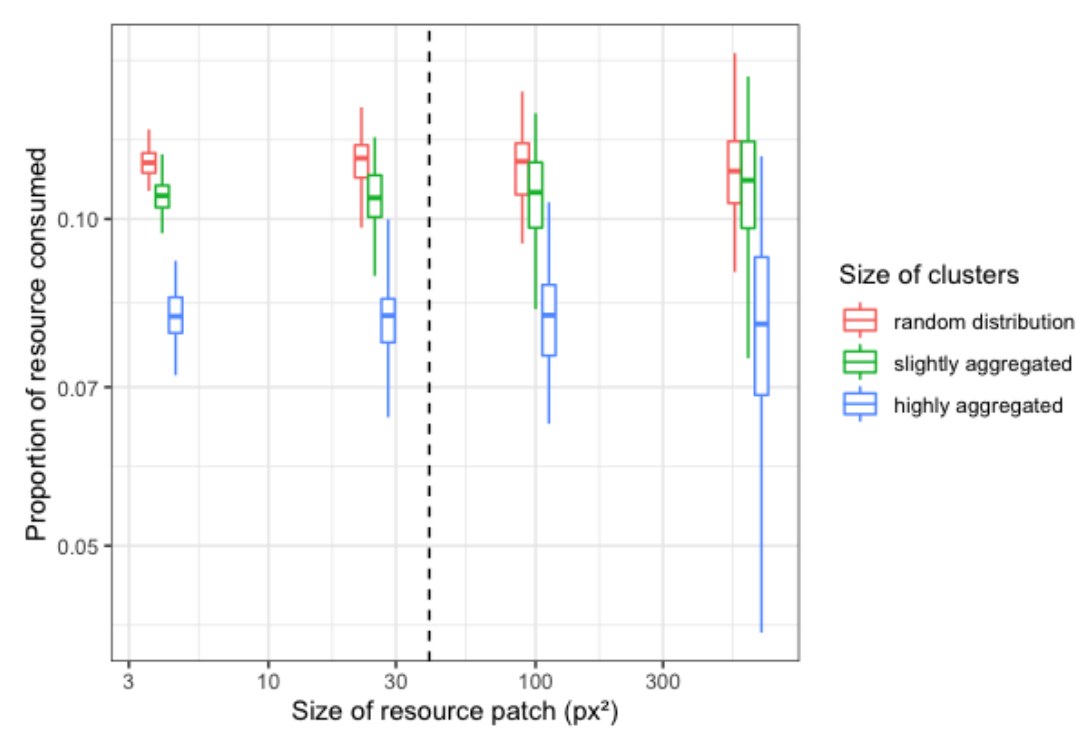

Figure 6 Proportion of resource consumed as a function of decomposer and resource spatial distribution in the case of decomposers acquiring resources within a disc with a radius $r=40 p x$ and constant biomass (120 cells in the $100 x 100$ grid). Decomposers had different resource use profiles were as described in the body of the text. The cluster sizes were created using a clustered Matèrn distribution where the radius of the decomposer clusters (rc) was either 10px (highly clustered) or 25px (slightly clustered). Note that the scales of both axes are logarithmic.

\section{Conclusions}

A number of points emerge from this study. The first is that the access decomposers have to resources has a significant effect on resource use. In this model, decomposer access to resources,

475 represented here by the size of the decomposer discs, is the primary regulator of resource use (see differences in Fig 3 relative to differences in Figs 4-6). Whilst the conclusion seems to be a reasonable one - contact between decomposer or decomposer enzymes and resource is necessary for the resource to be used [59] - the extent to which access regulates the activity of decomposition would need to be tested. To do so requires that the properties of the system that affect decomposer access to resources

480 be better parametrised. The access is a function of a number of abiotic soil properties, including, but not limited to, pore structure, specific surface area, connectivity of the aqueous phase and the relative distributions of decomposers and resources. A better understanding of how these properties vary at scales relevant to microbial activity would further our capacity to identify more clearly where the 
relationship between bacterial communities and soil organic matter transformations lies [60].

485 Decomposer traits, such as substrate use rates (which affect the diffusional gradient of a substrate and the length of diffusion pathways) or carbon use efficiency (which would affect biomass production and subsequent substrate use), may also affect decomposer access to resources. Whilst we expect the effects of microbial traits to be smaller than those of the local environmental properties, this remains to be quantified. However, the incorporation of carbon use efficiency into $\mathrm{C}$ dynamics models [61]

490 may be a simple way of representing this access. Decomposer access to substrate in soil depends not only on bacterial decomposers but also on fungi. As already stated, fungi are primarily found in copiotrophic environments (litter layers and rhizosphere - [38]). In these environments, and others where there are sufficient resources to facilitate hyphal spread, fungi would be expected to have a homogenising or spatial averaging effect. This would occur because of their ability to overcome the

495 spatial separation between resources that might arise in spatially heterogeneous environments. It has also been suggested that fungi can transport bacterial cells (known as the "fungal highway" - [62]), which would clearly affect the potential of bacterial decomposers to explore heterogeneous space. However, the extent to which this occurs in natural soils is unclear and we have not included this aspect in the simulations. Fungal carbon use efficiency is also believed to be different from that of

500 bacteria (e.g. [63]), which would also affect decomposition rates and resource use, but this aspect is beyond the scope of this particular study.

The second point is that the interaction between the spatial distribution of resources and that of decomposers can result in completely different resource uses, even though the resource availabilities and decomposer capacities are the same. This point may help explain why organic matter can persist

505 in soil despite the wealth of decomposer communities.

The third point is that there was no apparent trade-off between growth, assumed here to be related to the number of $16 \mathrm{~S}$ rRNA gene copies per cell, and the range of degradation/utilisation/assimilation pathways. If anything, the relationship between 16S rRNA gene copies and the number of pathways was positive, suggesting that maintaining a relatively high number

510 of degradation/utilisation/assimilation pathways increases fitness in a heterogeneous environment like soil. Indeed, the spatial simulations confirmed this: in the absence of significant trade-offs, resource use by the community of generalists was far greater than that of the community of specialists.

\section{Acknowledgements}

515 The authors would like to thank two anonymous referees for the pertinent and constructive comments they made which contributed to improving the manuscript. The funding received for two projects - Project ANR- 15-CE01-0006-01 (“Soil $\mu$ 3D”) and PHC Amadeus project $\mathrm{N}^{\circ} 44098 \mathrm{VJ}$ is also acknowledged. 
1. Roesch LFW et al. 2007 Pyrosequencing enumerates and contrasts soil microbial diversity. The ISME Journal (doi:10.1038/ismej.2007.53)

2. Wertz S, Degrange V, Prosser JI, Poly F, Commeaux C, Freitag T, Guillaumaud N, Roux XL. 2006 Maintenance of soil functioning following erosion of microbial diversity. Environmental Microbiology 8, 2162-2169. (doi:10.1111/j.1462-2920.2006.01098.x)

3. Prosser JI et al. 2007 The role of ecological theory in microbial ecology. Nature Reviews Microbiology 5, 384-392.

4. Loreau M. 2001 Microbial Diversity, Producer-Decomposer Interactions And Ecosystem Processes: A Theoretical Model. Proc R Soc Lond B 268, 303-309.

(doi:10.1098/rspb.2000.1366)

5. Fontaine S, Barot S. 2005 Size and functional diversity of microbe populations control plant persistence and long-term soil carbon accumulation. Ecol.Lett. 8, 1075-1087.

6. Loreau M. 1998 Biodiversity and ecosystem functioning: A mechanistic model. Proceedings of the National Academy of Sciences , 5632-5636.

7. Pfeiffer T, Schuster S, Bonhoeffer S. 2001 Cooperation and competition in the evolution of ATP-producing pathways. Science 292, 504-507.

8. Allison SD. 2012 A trait-based approach for modelling microbial litter decomposition. Ecol Lett 15, 1058-1070. (doi:10.1111/j.1461-0248.2012.01807.x)

9. Malik AA, Martiny JBH, Brodie EL, Martiny AC, Treseder KK, Allison SD. 2019 Defining trait-based microbial strategies with consequences for soil carbon cycling under climate change. ISME J (doi:10.1038/s41396-019-0510-0)

10. Nunan N. 2017 The microbial habitat in soil: Scale, heterogeneity and functional consequences. Journal of Plant Nutrition and Soil Science 180, 425-429. (doi:10.1002/jpln.201700184)

11. Egli T. 1995 The ecological and physiological significance of the growth of heterotrophic microorganisms with mixtures of substrates. In Advances in Microbial Ecology (ed JG Jones), pp. 305-386. Boston, MA: Springer US. (doi:10.1007/978-1-4684-7724-5_8)

12. Treves DS, Xia B, Zhou J, Tiedje JM. 2003 A two-species test of the hypothesis that spatial isolation influences microbial diversity in soil. Microb.Ecol. 45, 20-28.

13. Young IM, Crawford JW. 2004 Interactions and self-organization in the soil-microbe complex. Science 304, 1634-1637.

14. Or D, Smets BF, Wraith JM, Dechesne A, Friedman SP. 2007 Physical constraints affecting bacterial habitats and activity in unsaturated porous media - a review. Advances in Water Resources 30, 1505-1527.

15. Carson JK, Gonzalez-Quinones V, Murphy DV, Hinz C, Shaw JA, Gleeson DB. 2010 Low Pore Connectivity Increases Bacterial Diversity in Soil. Applied and Environmental Microbiology 76, 3936-3942. (doi:10.1128/AEM.03085-09) 
16. Dechesne A, Or D, Smets BF. 2008 Limited diffusive fluxes of substrate facilitate coexistence of two competing bacterial strains. FEMS Microbiol.Ecol. 64, 1-8.

17. Zhou JZ, Xia BC, Treves DS, Wu LY, Marsh TL, O’Neill RV, Palumbo AV, Tiedje JM. 2002 Spatial and resource factors influencing high microbial diversity in soil. Appl.Envron.Microbiol. 68, 326-334.

18. Long T, Or D. 2005 Aquatic habitats and diffusion constraints affecting microbial coexistence in unsaturated porous media. Water Resour.Res. 41.

19. Lane N, Martin W. 2010 The energetics of genome complexity. Nature 467, 929-934. (doi:10.1038/nature09486)

20. Lynch M, Marinov GK. 2015 The bioenergetic costs of a gene. Proc Natl Acad Sci USA, 201514974. (doi:10.1073/pnas.1514974112)

21. Scott M, Gunderson CW, Mateescu EM, Zhang Z, Hwa T. 2010 Interdependence of Cell Growth and Gene Expression: Origins and Consequences. Science 330, 1099-1102. (doi:10.1126/science.1192588)

22. Smith DR, Chapman MR. 2010 Economical Evolution: Microbes Reduce the Synthetic Cost of Extracellular Proteins. mBio 1, e00131-10. (doi:10.1128/mBio.00131-10)

23. Akashi H, Gojobori T. 2002 Metabolic efficiency and amino acid composition in the proteomes of Escherichia coli and Bacillus subtilis. PNAS 99, 3695-3700. (doi:10.1073/pnas.062526999)

24. Baudouin-Cornu P. 2001 Molecular Evolution of Protein Atomic Composition. Science 293, 297-300. (doi:10.1126/science.1061052)

25. Dekel E, Alon U. 2005 Optimality and evolutionary tuning of the expression level of a protein. Nature 436, 588-592. (doi:10.1038/nature03842)

26. Traving SJ, Thygesen UH, Riemann L, Stedmon CA. 2015 A Model of Extracellular Enzymes in Free-Living Microbes: Which Strategy Pays Off? Appl. Environ. Microbiol. 81, 7385-7393. (doi:10.1128/AEM.02070-15)

27. Hense BA, Kuttler C, Müller J, Rothballer M, Hartmann A, Kreft J-U. 2007 Does efficiency sensing unify diffusion and quorum sensing? Nature Reviews Microbiology 5, 230-239. (doi:10.1038/nrmicro1600)

28. Gao S, DeLuca TH. 2019 Use of microdialysis to assess short-term soil soluble N dynamics with biochar additions. Soil Biology and Biochemistry 136, 107512.

(doi:10.1016/j.soilbio.2019.06.008)

29. Leitner S, Homyak PM, Blankinship JC, Eberwein J, Jenerette GD, Zechmeister-Boltenstern S, Schimel JP. 2017 Linking NO and N 2 O emission pulses with the mobilization of mineral and organic N upon rewetting dry soils. Soil Biology and Biochemistry 115, 461-466. (doi:10.1016/j.soilbio.2017.09.005)

30. Raynaud X, Nunan N. 2014 Spatial Ecology of Bacteria at the Microscale in Soil. PLoS ONE 9, e87217. (doi:10.1371/journal.pone.0087217)

31. Roth V-N, Dittmar T, Gaupp R, Gleixner G. 2014 Ecosystem-Specific Composition of Dissolved Organic Matter. Vadose Zone Journal 13, 0. (doi:10.2136/vzj2013.09.0162) 
32. Hooper DU et al. 2005 Effects of biodiversity on ecosystem functioning: a consensus of current knowledge. Ecological Monographs 75, 3-35. (doi:10.1890/04-0922)

33. Taylor JR, Stocker R. 2012 Trade-Offs of Chemotactic Foraging in Turbulent Water. Science 338, 675-679. (doi:10.1126/science.1219417)

34. Stocker R. 2012 Marine Microbes See a Sea of Gradients. Science 338, 628-633. (doi:10.1126/science.1208929)

35. Aleklett K, Kiers ET, Ohlsson P, Shimizu TS, Caldas VE, Hammer EC. 2018 Build your own soil: exploring microfluidics to create microbial habitat structures. ISME J 12, 312-319. (doi:10.1038/ismej.2017.184)

36. Dechesne A, Wang G, Gulez G, Or D, Smets BF. 2010 Hydration-controlled bacterial motility and dispersal on surfaces. Proceedings of the National Academy of Sciences 107, 14369-14372. (doi:10.1073/pnas.1008392107)

37. Bailey VL, Smith JL, Bolton H. 2002 Fungal-to-bacterial ratios in soils investigated for enhanced C sequestration. Soil Biology and Biochemistry 34, 997-1007. (doi:10.1016/S00380717(02)00033-0)

38. Frostegård A, Bååth E. 1996 The use of phospholipid fatty acid analysis to estimate bacterial and fungal biomass in soil. Biol Fert Soils 22, 59-65. (doi:10.1007/BF00384433)

39. German DP, Chacon SS, Allison SD. 2011 Substrate concentration and enzyme allocation can affect rates of microbial decomposition. Ecology 92, 1471-1480. (doi:10.1890/10-2028.1)

40. Roberson EB, Firestone MK. 1992 Relationship between Desiccation and Exopolysaccharide Production in a Soil Pseudomonas sp. Appl. Environ. Microbiol. 58, 1284-1291.

41. Romaní AM, Fund K, Artigas J, Schwartz T, Sabater S, Obst U. 2008 Relevance of Polymeric Matrix Enzymes During Biofilm Formation. Microb Ecol 56, 427-436. (doi:10.1007/s00248007-9361-8)

42. Costa OYA, Raaijmakers JM, Kuramae EE. 2018 Microbial Extracellular Polymeric Substances: Ecological Function and Impact on Soil Aggregation. Front. Microbiol. 9, 1636. (doi:10.3389/fmicb.2018.01636)

43. Jayathilake PG, Jana S, Rushton S, Swailes D, Bridgens B, Curtis T, Chen J. 2017 Extracellular Polymeric Substance Production and Aggregated Bacteria Colonization Influence the Competition of Microbes in Biofilms. Front. Microbiol. 8, 1865. (doi:10.3389/fmicb.2017.01865)

44. Lipson DA. 2015 The complex relationship between microbial growth rate and yield and its implications for ecosystem processes. Front. Microbiol. 6. (doi:10.3389/fmicb.2015.00615)

45. Caspi R et al. 2018 The MetaCyc database of metabolic pathways and enzymes. Nucleic Acids Res 46, D633-D639. (doi:10/ggbrws)

46. Roller BRK, Stoddard SF, Schmidt TM. 2016 Exploiting rRNA operon copy number to investigate bacterial reproductive strategies. Nat Microbiol 1, 16160.

(doi:10.1038/nmicrobiol.2016.160) 
47. Delgado-Baquerizo M, Oliverio AM, Brewer TE, Benavent-González A, Eldridge DJ, Bardgett RD, Maestre FT, Singh BK, Fierer N. 2018 A global atlas of the dominant bacteria found in soil. Science 359, 320-325. (doi:10.1126/science.aap9516)

48. Kielak AM, Barreto CC, Kowalchuk GA, van Veen JA, Kuramae EE. 2016 The Ecology of Acidobacteria: Moving beyond Genes and Genomes. Front. Microbiol. 7.

(doi:10.3389/fmicb.2016.00744)

49. Barberán A, Bates ST, Casamayor EO, Fierer N. 2012 Using network analysis to explore cooccurrence patterns in soil microbial communities. The ISME Journal 6, 343-351.

(doi:10.1038/ismej.2011.119)

50. Stoddard SF, Smith BJ, Hein R, Roller BRK, Schmidt TM. 2015 rnDB: improved tools for interpreting rRNA gene abundance in bacteria and archaea and a new foundation for future development. Nucleic Acids Research 43, D593-D598. (doi:10.1093/nar/gku1201)

51. Gyorfy Z, Draskovits G, Vernyik V, Blattner FF, Gaal T, Posfai G. 2015 Engineered ribosomal RNA operon copy-number variants of E. coli reveal the evolutionary trade-offs shaping rRNA operon number. Nucleic Acids Research 43, 1783-1794. (doi:10.1093/nar/gkv040)

52. Li J et al. 2019 Predictive genomic traits for bacterial growth in culture versus actual growth in soil. ISME J 13, 2162-2172. (doi:10.1038/s41396-019-0422-z)

53. Oyewole OA, Inselsbacher E, Näsholm T. 2014 Direct estimation of mass flow and diffusion of nitrogen compounds in solution and soil. New Phytologist 201, 1056-1064.

(doi:10.1111/nph.12553)

54. Stoyan D. 1998 Random Sets: Models and Statistics. International Statistical Review / Revue Internationale de Statistique 66, 1. (doi:10.2307/1403654)

55. Molchanov IS. 1995 Statistics of the Boolean model: from the estimation of means to the estimation of distributions. Advances in Applied Probability 27, 25.

56. Illian J, editor. 2008 Statistical analysis and modelling of spatial point patterns. Chichester, England; Hoboken, NJ: John Wiley.

57. Hassink J. 1994 Effects of soil texture and grassland management on soil organic $\mathrm{C}$ and $\mathrm{N}$ and rates of C and N mineralization. Soil Biology and Biochemistry 26, 1221-1231. (doi:10.1016/0038-0717(94)90147-3)

58. Kuzyakov Y, Blagodatskaya E. 2015 Microbial hotspots and hot moments in soil: Concept \& review. Soil Biology and Biochemistry 83, 184-199. (doi:10.1016/j.soilbio.2015.01.025)

59. Dignac M-F et al. 2017 Increasing soil carbon storage: mechanisms, effects of agricultural practices and proxies. A review. Agronomy for Sustainable Development 37. (doi:10.1007/s13593-017-0421-2)

60. Baveye PC et al. 2018 Emergent Properties of Microbial Activity in Heterogeneous Soil Microenvironments: Different Research Approaches Are Slowly Converging, Yet Major Challenges Remain. Frontiers in Microbiology 9. (doi:10.3389/fmicb.2018.01929)

61. Allison SD, Wallenstein MD, Bradford MA. 2010 Soil-carbon response to warming dependent on microbial physiology. Nature Geoscience 3, 336-340. (doi:10.1038/ngeo846) 
62. Kohlmeier S, Smits THM, Ford RM, Keel C, Harms H, Wick LY. 2005 Taking the Fungal Highway: Mobilization of Pollutant-Degrading Bacteria by Fungi. Environ. Sci. Technol. 39, 4640-4646. (doi:10.1021/es047979z)

63. Keiblinger KM et al. 2010 The effect of resource quantity and resource stoichiometry on microbial carbon-use-efficiency: Resource quantity/quality drives microbial C-use-efficiency. FEMS Microbiology Ecology, no-no. (doi:10.1111/j.1574-6941.2010.00912.x) 